\title{
Presentazione della Fondazione Simone Cesaretti
}

\section{Scopo della Fondazione}

Il 6 Novembre 2007, per iniziativa della famiglia Cesaretti, è stata costituita la Fondazione Simone Cesaretti, che opera senza fini di lucro e ha durata illimitata. Alla Fondazione è stata riconosciuta la personalità giuridica di diritto privato con il Decreto Dirigenziale n. 334/2008 della Regione Campania ed è stata iscritta al n. 230 nel Registro delle persone giuridiche, istituito presso la Regione Campania - Settore Rapporti con Province, Comuni, CC.MM..

La Fondazione, attraverso la costituzione di network tra Università, centri di ricerca, Enti pubblici e privati, società di comunicazione, ha lo scopo di contribuire mediante studi, ricerche, iniziative di formazione e informazione, all'approfondimento delle conoscenze concernenti le tematiche della sostenibilità dello sviluppo, analizzata nelle sue diverse dimensioni: ambientale, economica, territoriale, sociale e generazionale. In particolare, la Fondazione intende dedicare un'attenzione specifica alla dimensione generazionale quale elemento propulsivo dello sviluppo sostenibile. In relazione a tali tematiche, la Fondazione intende porre in essere adeguate strategie di sensibilizzazione e comunicazione, per favorire la promozione di una cultura integrata della sostenibilità.

\section{Consiglio di Amministrazione}

Sono organi istituzionali della Fondazione il Presidente, nella persona di Gian Paolo Cesaretti, per i primi cinque anni, l'Assemblea dei Soci Fondatori, il Consiglio di Amministrazione, il Collegio dei Saggi e gli altri organi necessari al funzionamento della stessa, definiti nello Statuto.

Tab. 1 - Consiglio di Amministrazione

\begin{tabular}{|c|}
\hline CONSIGLIO DI AMMINISTRAZIONE \\
\hline PRESIDENTE: Gian Paolo Cesaretti \\
\hline VICEPRESIDENTE: Augusto Marinelli \\
\hline CONSIGLIERE: Simona Bizzarri \\
\hline
\end{tabular}

Rivista di Studi sulla Sostenibilità, n. 1/2011 


\section{Collegio dei Saggi}

Il ruolo di fornire l'indirizzo scientifico-culturale alle attività programmate dalla Fondazione è garantito dall'istituzione del Collegio dei Saggi, organo di primaria importanza, il cui Presidente Onorario è la Prof.ssa Rita Levi Montalcini, premio Nobel per la medicina nel 1986 e socia nazionale dell'Accademia dei Lincei. La presidenza è stata affidata al Prof. Adriano Giannola, Presidente dell'Istituto Banco di Napoli -Fondazione, mentre la vice presidenza alla Prof.ssa Eva Pföstl, Coordinatrice scientifica dell'area giuridico-economica dell'Istituto di Studi Politici "S. Pio V".

Tab. 2 - Collegio dei Saggi

\begin{tabular}{|c|c|}
\hline \multicolumn{2}{|c|}{ PRESIDENTE ONORARIO } \\
\hline $\begin{array}{l}\text { Prof.ssa Rita Levi } \\
\text { Montalcini }\end{array}$ & $\begin{array}{l}\text { Premio Nobel per la medicina nel } 1986 \text { e socia naziona- } \\
\text { le dell'Accademia dei Lincei }\end{array}$ \\
\hline \multicolumn{2}{|l|}{ PRESIDENTE } \\
\hline $\begin{array}{l}\text { Prof. Adriano Gian- } \\
\text { nola }\end{array}$ & Presidente dell'Istituto Banco di Napoli-Fondazione \\
\hline \multicolumn{2}{|c|}{ VICE PRESIDENTE } \\
\hline Prof. ssa Eva Pföstl & $\begin{array}{l}\text { Coordinatrice scientifica area giuridico-economica } \\
\text { dell'Istituto di Studi Politici "S. Pio V" }\end{array}$ \\
\hline \multicolumn{2}{|c|}{ MEMBRI DEL COLLEGIO DEI SAGGI } \\
\hline Prof. Gennaro Ferrara & Università degli Studi di Napoli "Parthenope" \\
\hline $\begin{array}{l}\text { Prof.ssa Marisa Fer- } \\
\text { rari Occhionero }\end{array}$ & $\begin{array}{l}\text { Delegato del Rettore per le Pari Opportunità } \\
\text { Università degli Studi di Roma "La Sapienza" }\end{array}$ \\
\hline $\begin{array}{l}\text { Prof. Sebastiano } \\
\text { Maffettone }\end{array}$ & $\begin{array}{l}\text { Preside della Facoltà di Scienze Politiche della Luiss } \\
\text { Guido Carli e Professore Ordinario di Filosofia Politica }\end{array}$ \\
\hline $\begin{array}{l}\text { Dott. Maurizio Mari- } \\
\text { nella }\end{array}$ & Imprenditore \\
\hline $\begin{array}{l}\text { Prof. Augusto Mari- } \\
\text { nelli }\end{array}$ & Università degli Studi di Firenze \\
\hline $\begin{array}{l}\text { Prof. Mario Morcel- } \\
\text { lini }\end{array}$ & $\begin{array}{l}\text { Preside della Facoltà di Scienze della Comunicazione- } \\
\text { Università degli Studi di Roma "La Sapienza" }\end{array}$ \\
\hline Dott. Erwin Northoff & $\begin{array}{l}\text { Ufficio Stampa, Food and Agriculture Organization of } \\
\text { the United Nations }\end{array}$ \\
\hline Prof. Claudio Quintano & Rettore dell'Università degli Studi di Napoli “Parthenope" \\
\hline Dott. Stefano Ribaldi & Dirigente RAI \\
\hline $\begin{array}{l}\text { Prof. Wolfgang } \\
\text { Sachs }\end{array}$ & Senior Research Fellow al Wuppertal Institut \\
\hline $\begin{array}{l}\text { Prof. Ranabir Sa- } \\
\text { maddar }\end{array}$ & $\begin{array}{l}\text { Direttore del Calcutta Reaserch Centre of Human } \\
\text { Rights. }\end{array}$ \\
\hline
\end{tabular}




\section{Partecipanti Istituzionali}

La Fondazione Simone Cesaretti ha previsto nel suo statuto la possibilità di riconoscere la qualifica di Partecipante a coloro che, condividendo le sue finalità, la sostengono e contribuiscono anche in via non continuativa alla realizzazione dei suoi scopi mediante contributi in denaro, in attività $\mathrm{o}$ in beni o servizi materiali o immateriali, o in altre forme ritenute idonee. Agli enti e le amministrazioni pubbliche, alle persone fisiche e giuridiche, pubbliche o private, che collaborano stabilmente alle attività la Fondazione attribuisce la qualifica di Partecipante Istituzionale. Inoltre, a coloro che abbiano contribuito in maniera rilevante e significativa con mezzi e risorse al suo sviluppo e ai suoi obiettivi, la Fondazione conferisce la qualifica di Partecipante Onorario.

\section{Tab. 3 - Partecipanti istituzionali}

\begin{tabular}{|l|}
\hline \multicolumn{1}{|c|}{ PARTECIPANTI ISTITUZIONALI } \\
\hline Academia de Studii Ecomomice Bucaresti (Feam) - Bucarest \\
\hline Banca del Sud - Napoli \\
\hline Camera di Commercio - Napoli \\
\hline Consiglio per la Ricerca e la Sperimentazione in Agricoltura (CRA) - Roma \\
\hline $\begin{array}{l}\text { Centro Regionale di Educazione ed Informazione Ambientale (CREIA) - Fondi } \\
\text { (LT) }\end{array}$ \\
\hline Confederazione Libere Associazioni Artigiane Italiane (CLAAI) - Napoli \\
\hline Institut National de la Recherche Agronomique (INRA) - Paris \\
\hline Istituto Banco di Napoli - Fondazione \\
\hline Istituto Nazionale di Economia Agraria (INEA) - Roma \\
\hline Istituto Nazionale di Ricerca per gli Alimenti e la Nutrizione (INRAN) - Roma \\
\hline Istituto Nazionale di Statistica (ISTAT) - Roma \\
\hline $\begin{array}{l}\text { Istituto Nazionale di Studi di Agribusiness e Sostenibilità (INAS) Firenze - Con- } \\
\text { sorzio Interuniversitario tra le Università di Firenze, Verona e Napoli "Partheno- } \\
\text { pe” }\end{array}$ \\
\hline $\begin{array}{l}\text { Libera Università Internazionale degli Studi Sociali Guido Carli, Roma - Dipar- } \\
\text { timento di Scienze storiche e socio-politiche }\end{array}$ \\
\hline Monumenti Naturali - Parco Naturale Regionale Monti Ausoni - Lazio \\
\hline $\begin{array}{l}\text { Università degli Studi di Firenze - Dipartimento di Economia, Ingegneria, } \\
\text { Scienze e Tecnologie Agrarie e Forestali (DEISTAF) }\end{array}$ \\
\hline Università Cà Foscari di Venezia - Dipartimento di Statistica \\
\hline $\begin{array}{l}\text { Università degli Studi del Sannio - Dipartimento di Analisi dei sistemi economi- } \\
\text { ci e sociali }\end{array}$ \\
\hline
\end{tabular}


Università degli Studi della Basilicata - Dipartimento Tecnico-Economico per la gestione del Territorio Agricolo-Forestale (DITEC)

Università degli Studi di Cassino - Dipartimento Istituzioni, Metodi Quantitativi e Territorio

Università degli Studi di Catania - Dipartimento di Scienze Economico-Agrarie ed Estimative (DISEAE)

Università degli Studi di Firenze - Centro universitario di ricerca e formazione per lo sviluppo competitivo delle imprese del settore vitivinicolo italiano (UniCeSV)

Università degli Studi di Milano - Dipartimento di Economia e Politica Agraria, Agro-alimentare e Ambientale

Università degli Studi di Napoli "Parthenope"- Dipartimento di Studi Economici

Università degli Studi di Verona - Dipartimento di Scienze, Tecnologie e Mercati della vite e del Vino (Di Ste MeV)

Università di Roma La Sapienza - Centro di Ricerca "Studi per lo Sviluppo" (SPES)

\section{Strumenti operativi della Fondazione}

Per il raggiungimento dei propri fini, oltre alla Rivista di Studi sulla sostenibilità, la Fondazione si avvale di strumenti operativi ritenuti di strategica rilevanza, quali:

- una Collana di Studi sulla sostenibilità, quale strumento formativodivulgativo, che si rivolge ad un pubblico ampio.

- un Portale della Sostenibilità, che rappresenta un centro virtuale di dibattito ed un contenitore di contributi innovativi ed originali, mediante il quale comunicare in maniera diffusa ed interattiva le questioni ed i temi connessi alla sostenibilità dello sviluppo.

- un Forum sulla sostenibilità dello sviluppo umano: punto di riferimento stabile nel dibattito sulla sostenibilità dello sviluppo umano; un momento importante intorno a cui aggregare le migliori menti, competenze ed energie al fine di discutere, analizzare e approfondire in maniera multidimensionale ed interdisciplinare le questioni connesse alla sostenibilità dello sviluppo umano e di ideare soluzioni innovative.

\section{Sistema formativo}

La Fondazione Simone Cesaretti intende porre in essere un Sistema Formativo, fondato su una rete di relazioni e partnership che coinvolge diverse Istituzioni formative (Università; Enti di formazione; Istituti scolastici; Associazioni culturali ecc.). 
In tal senso la Fondazione Simone Cesaretti ha identificato un ventaglio di modalità mediante le quali proporre la propria offerta formativa sia a giovani studenti che a professionisti che, a vario titolo, sono già impegnati nel mondo del lavoro. Tali modalità, inoltre, includono non solo l'alta formazione mediante l'attivazione di Master, Seminari e Workshop, Borse di studio e Stage in Fondazione, ma anche la realizzazione di Progetti didattici ed educativi destinati alle scuole secondarie e agli Istituti formativi.

\section{Web Project}

La Fondazione Simone Cesaretti favorisce l'ideazione e la realizzazione di progetti web volti a promuovere la cultura globale della sostenibilità e ad avvicinare i giovani attraverso l'interazione virtuale.

Il Portale della Sostenibilità, www.portaledellasostenibilita.it, rappresenta lo strumento ideato per assicurare, attraverso un sistema di comunicazione integrata, l'offerta di un patrimonio conoscitivo sui principali temi connessi allo sviluppo e all'avanzamento nella promozione di una cultura globale della sostenibilità.

La Fondazione, consapevole della centralità delle Aree Marine Protette quali strumenti privilegiati nell'implementazione di strategie di sviluppo sostenibile, ha realizzato il sito Aree Marine Protette, www.areemarineprotette.it.

Attualmente, la Fondazione sta progettando il portale dell'Osservatorio sulle identità culturali territoriali ed il portale Parchi e Riserve naturali quali strumenti ideali per diffondere tra i giovani la conoscenza delle identità territoriali e per sperimentare con essi l'interazione virtuale sulla centralità dei parchi e delle riserve naturali nel perseguimento della sostenibilità. 